\title{
Magnetic Collapse in Electroweak Plasma
}

\author{
A. Perez Martinez ${ }^{a}$, H. Perez Rojas ${ }^{b, c *}$ \\ Address \\ ${ }^{a}$ Depto. de Fisica.Universidad Nacional de Colombia, Sede Medellin \\ ${ }^{b}$ ICIMAF, Calle E No. 309, 10400 La Habana, Cuba \\ ${ }^{c}$ International Centre for Theoretical Physics \\ P.O. Box 586, Strada Costiera 11, 34100 Trieste, Italy \\ E-mail: 'amperez@perseus unalmed.edu.co and hugo@cidet.icmf.inf.cu
}

ABstract: We discuss the thermodynamics of degenerate electron and charged vector boson gases in very intense magnetic fields. In degenerate conditions of the electron gas, there is a dependence of density with regard to field intensity for which the pressure transverse to the magnetic field vanishes, leading to a transverse collapse. For $W$ bosons an instability arises because the magnetization diverges at the critical field $B_{c}=M_{W}^{2} / e$. If the magnetic field is self-consistently maintained, either the transverse collapse occurs at fields of order $2 B_{c} / 3$, or the instability is avoided by some cooling mechanism.

\section{Introduction}

Magnetic fields of order $10^{20} \mathrm{G}$ and larger have been suggested to exist in the cores of neutron stars [2] The standard electroweak theory establishes a limit on the magnetic field, the critical upper bound being $B_{c}=M_{W}^{2} / e \simeq 1.06$. $10^{24} \mathrm{G}$. That instability can be seen from the expression for the $W^{ \pm}$ground state energy $\epsilon_{0 q}=$ $\sqrt{M_{W}^{2}-e B}$, which becomes purely imaginary for $B>B_{c}$. Fields of order $B_{c}$ may have been created at the electroweak phase transition (see [ind [3i]). In astrophysics, also the critical field $B_{c^{\prime}}=$ $m_{e}^{2} / e \simeq 4.41 \cdot 10^{13} \mathrm{G}$ is relevant.

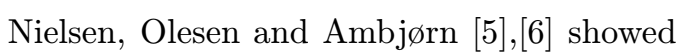
by considering static solutions of the equations of motion of the electroweak gauge bosons ( $W$ and $Z$ ) that the vacuum possesses the properties of a ferromagnet or an antiscreening superconductor for $B \sim B_{c}$. It thus seems relevant to study the electroweak medium in a strong magnetic field of the order of the critical magnetic fields. The implications of these results for astroparticle physics and cosmology are expected to be interesting. Here we shall calculate the

*This paper is based on ref. [1], written in collaboration with M. Chaichian, C. Montonen and S. Masood magnetization due to the charged leptons and intermediate vector bosons in the standard model.

To start with we shall write some basic formulae. The partition function $\mathcal{Z}$ which is obtained from the density matrix leads to the thermodynamic potential $\Omega=-T \ln \mathcal{Z}$ involving the contributions from the species of leptons and quarks involved, which are considered to be in chemical equilibrium among themselves through the boson fields, described by equations among their chemical potentials [8, of the sort $\mu_{W^{+}}=\mu_{\nu}+\mu_{e^{+}}$, $\mu_{d_{L}}+\mu_{W^{+}}=\mu_{u_{L}}, \mu_{e^{+}, W^{+}}+\mu_{e^{-}, W^{-}}=0$. From this general thermodynamical potential we will choose the electron and $W$ sectors exhibiting interesting effects in the astrophysical and cosmological scenarios respectively in the presence of extremely strong magnetic fields $\left(B \sim B_{c^{\prime}}\right.$ and $\left.B \sim B_{c}\right)$.

\section{The thermodynamical potential}

It is well known that the denser the Fermi gas,

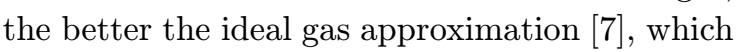
is valid in presence of an external magnetic field. In our case, the ideal gas thermodynamical potential per unit volume of the electron-positron 
sector is $\Omega_{e}=\Omega_{s e}+\Omega_{0 e}$, where

$$
\Omega_{s e}=-\frac{e B}{4 \pi^{2} \beta} \sum_{n=0}^{\infty} a_{n} \int_{-\infty}^{\infty} d p_{3} \ln f_{+}^{e} f_{-}^{e}
$$

where $f_{ \pm}^{e}=\left(1+e^{-\left(E_{q} \mp \mu_{e}\right) \beta}\right)$ and the sum extends over all Landau quantum numbers $E_{q}=$ $\sqrt{p_{3}^{2}+m_{e}^{2}+2 e B n}$, the degeneracy factor is $a_{n}=$ $2-\delta_{0 n}$, and $\beta=T^{-1}$. (Actually, this degeneracy dissapears if we consider the anomalous magnetic moment of electrons; it plays a significant role for fields $B \gg B_{c^{\prime}}$. For our present approximation, we will ignore it). For $W$ 's, we have $\Omega_{W}=\Omega_{s W}+\Omega_{0 W}$

$$
\begin{aligned}
\Omega_{s W}= & \frac{e B}{4 \pi^{2} \beta} \int_{-\infty}^{\infty} d p_{3} \ln f_{+}^{0 W} f_{-}^{0 W}+ \\
& \frac{e B}{4 \pi^{2} \beta} \sum_{n=0}^{\infty} b_{n} \int_{-\infty}^{\infty} d p_{3} \ln f_{+}^{W} f_{-}^{W}
\end{aligned}
$$

where $f_{ \pm}^{0 W}=\left(1-e^{-\left(\epsilon_{0 q} \mp \mu_{W}\right) \beta}\right), f_{ \pm}^{W}=(1-$ $\left.e^{-\left(\epsilon_{q} \mp \mu_{W}\right) \beta}\right)$, again we sum over all Landau quantum numbers and the degeneracy factor is $b_{n}=$ $3-\delta_{0 n}$, with $\epsilon_{0 q}=\sqrt{p_{3}^{2}+M_{W}^{2}-e B}$, and $\epsilon_{q}=$ $\sqrt{p_{3}^{2}+M_{W}^{2}+2 e B\left(n+\frac{1}{2}\right)}$.

The Euler-Heisenberg vacuum terms are, for the electron-positron field,

$$
\Omega_{0 e}=\frac{e^{2} B^{2}}{8 \pi^{2}} \int_{0}^{\infty} e^{-m_{e}^{2} x / e B} \frac{E(x) d x}{x},
$$

where $E(x)=x^{-1} \operatorname{coth} x-x^{-2}-1 / 3$. For the charged gauge bosons one obtains,

$$
\Omega_{0 W}=-\frac{e^{2} B^{2}}{16 \pi^{2}} \int_{0}^{\infty} e^{-M_{W}^{2} x / e B} \frac{W(x) d x}{x^{2}}
$$

where $W(x)=(1+2 \cosh 2 x) / \sinh x-3 x^{-1}-$ $7 x / 2$. We observe that $(2.4)$ diverges at $B=B_{c}$, leading to a vacuum instability.

The mean density of particles minus antiparticles (average charge divided by $e$ ) is given by $N_{e, W}=-\partial \Omega_{e, W} / \partial \mu_{e, W}$. We assume that there is always a background charge of opposite sign, to preserve electrical neutrality. We have

$$
N_{e}=\frac{e B}{4 \pi^{2}} \sum_{0}^{\infty} a_{n} \int_{-\infty}^{\infty} d p_{3}\left(n_{e}^{+}-n_{e}^{-}\right)
$$

where $n_{e}^{ \pm}=\left[\exp \left(E_{q} \mp \mu_{e}\right) \beta+1\right]^{-1}$.
In the degenerate limit one gets

$$
N_{e}=\frac{e B}{2 \pi^{2}} \sum_{0}^{n_{\mu}} a_{n} \sqrt{\mu_{e}^{2}-m^{2}-2 e B n},
$$

where the integer $n_{\mu}=I\left[\left(\mu_{e}^{2}-m^{2}\right) / 2 e B\right]$.

For $W$-s,

$$
\begin{aligned}
N_{W}= & \frac{e B}{4 \pi^{2}} \int_{-\infty}^{\infty} d p_{3}\left(n_{0 p}^{+}-n_{0 p}^{-}\right)+ \\
& \frac{e B}{4 \pi^{2}} \sum_{0}^{\infty} b_{n} \int_{-\infty}^{\infty} d p_{3}\left(n_{p}^{+}-n_{p}^{-}\right)
\end{aligned}
$$

where we define $n_{0 p}^{ \pm}=\left[\exp \left(\epsilon_{0 q} \mp \mu_{W}\right) \beta-1\right]^{-1}$, $n_{p}^{ \pm}=\left[\exp \left(\epsilon_{q} \mp \mu_{W}\right) \beta-1\right]^{-1}$.

The magnetization is given by the contribution of electrons and charged vector bosons. It depends on the density of particles plus antiparticles, and it is,

$$
\mathcal{M}_{W, e}=-\partial \Omega_{W, e} / \partial B
$$

where (by calling $\mathcal{M}_{0 e, 0 W}=-\partial \Omega_{0 e, 0 W} / \partial B$,

$$
\begin{aligned}
\mathcal{M}_{e}= & -\frac{\Omega_{s e}}{B}-\frac{e}{4 \pi^{2}} \sum_{0}^{\infty} a_{n} \int_{-\infty}^{\infty} d p_{3} \frac{e B n}{E_{q}}\left(n_{e}^{+}+n_{e}^{-}\right) \\
& +\mathcal{M}_{0 e}
\end{aligned}
$$

and in the degenerate limit $\left[\begin{array}{l}\text { ind } \\ 1\end{array}\right]$

$$
\begin{aligned}
\mathcal{M}_{e}= & \frac{e}{4 \pi^{2}} \sum_{0}^{n_{\mu}} a_{n}\left[\left(\mu_{e} \sqrt{\mu_{e}^{2}-m^{2}-2 e B n}-\right.\right. \\
& \left.\left(m^{2}+4 e B n\right) \ln \frac{\mu_{e}+\sqrt{\mu_{e}^{2}-m^{2}-2 e B n}}{\sqrt{m^{2}+2 e B n}}\right] \\
& +\mathcal{M}_{0 e},
\end{aligned}
$$

and

$$
\begin{aligned}
\mathcal{M}_{W}= & -\frac{\Omega_{W}}{B}+\frac{e^{2} B}{8 \pi^{2}}\left[\int_{-\infty}^{\infty} \frac{d p_{3}}{\epsilon_{q}^{0}}\left(n_{0 p}^{+}+n_{0 p}^{-}\right)\right]- \\
& \frac{e^{2} B}{4 \pi^{2}} \sum_{0}^{\infty} b_{n}\left(n+\frac{1}{2}\right)\left[\int_{-\infty}^{\infty} \frac{d p_{3}}{\epsilon_{q}}\left(n_{p}^{+}+n_{p}^{-}\right)\right] \\
& +\mathcal{M}_{0 W} .
\end{aligned}
$$




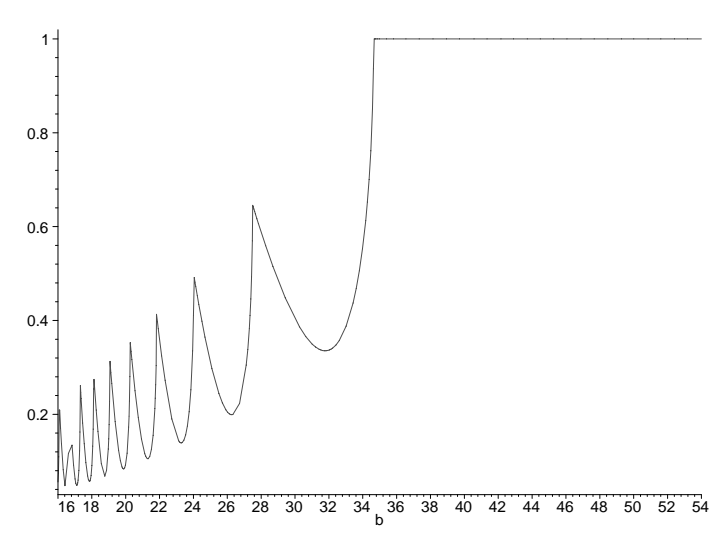

Figure 1: Oscillations of the relative magnetization $\mathcal{M} / \mathcal{M}_{0},\left(\mathcal{M}_{0}=e m_{e}^{2} / 4 \pi^{2}\right)$ as a function of the relative magnetic field $B / B_{c^{\prime}}$ up to saturation

\section{Equation of state}

At this point it is especially interesting to discuss the equation of state of the system. The total energy-momentum tensor, whose spatial diagonal components are the pressures along the coordinate axis, may be obtained by starting from the quantum statistical average of its standard fieldtheoretical expression $T_{\mu \nu}=\left\langle\mathcal{T}_{\mu \nu}\right\rangle_{s}$ where $\mathcal{T}_{\mu \nu}=$ $\frac{\partial \mathcal{L}}{\partial A_{\mu, \nu}} A_{\mu, \nu}-\delta_{\mu \nu} \mathcal{L}\left[10^{\prime}\right]$. Here $\mathcal{L}$ is the total Lagrangian, and after doing the statistical average, its place in the energy-momentum tensor is taken by $\Omega\left(\right.$ since $\left.\Omega=-\beta^{-1} \ln <e^{\int_{0}^{\beta} d x_{4} \int d^{3} x \mathcal{L}\left(x_{4}, \mathbf{x}\right)}>_{s}\right)$. The energy-momentum tensor is then,

$T_{\mu \nu}=\left(T \frac{\partial \Omega}{\partial T}+\mu \frac{\partial \Omega}{\partial \mu}\right) \delta_{4 \mu} \delta_{\nu 4}+4 F_{\mu \rho} F_{\nu \rho} \frac{\partial \Omega}{\partial F^{2}}-\delta_{\mu \nu} \Omega$,

where $F_{\mu \rho}$ is the electromagnetic field tensor average. For $F_{\mu \rho}=0$, (13.1) reproduces the usual zero field case $T_{\mu \nu}=p \delta_{\mu \nu}-(u+p) \delta_{4 \mu} \delta_{\nu 4}, u$ being the energy density. For the electrically charged particles, we obtain different equations of state for directions parallel and perpendicular to the magnetic field,

$$
p_{3}=-\Omega, \quad p_{\perp}=-\Omega-B \mathcal{M} .
$$

This anisotropy in the pressures $p_{3}, p_{\perp}$ leads to a magnetostriction effect in the quantum magnetized gas of charged particles. In classical theory (3.2) is the Maxwell stress tensor $\mathcal{M}<0$ and $p_{\perp}>p_{3}$, which produces a flattening effect in white dwarfs and neutron stars models [12],
[1] netic media also $\mathcal{M}<0$ leading again to a flattening effect. But for positive magnetization, the transverse pressure exerted by the charged particles is smaller than the longitudinal one by the amount $B \mathcal{M}$. This effect is actually present in the quantum vacuum in a magnetic field: by calculating the pressure of vacuum from the EulerHeisenberg formula, one obtains a negative transverse pressure, which can be attributed to the electron-positron virtual pairs. In a medium, the extreme case is found for magnetic fields, $e B \gg T^{2}$, when the electrons are confined to the Landau ground state $n=0$. (In what follows we will ignore the vacuum contribution to electronpositron pressure and magnetization, which is justified at the scale of densities and fields considered below). We have $\Omega_{e}=-B \mathcal{M}_{e}$ where,

$$
\begin{aligned}
\mathcal{M}_{e}= & \frac{e}{2 \pi^{2}}\left[\mu_{e} \sqrt{\mu_{e}^{2}-m^{2}}-\right. \\
& \left.m^{2} \ln \frac{\mu_{e}+\sqrt{\mu_{e}^{2}-m^{2}}}{m}\right]
\end{aligned}
$$

and $\mu_{e} \simeq \sqrt{\left(2 \pi^{2} N_{e} / e B\right)^{2}+m^{2}}, N_{e}$ being the electron density. As $\mu_{e}^{2}>m^{2}$, the expression (13.3) is always positive the system behaves as paramagnetic or ferromagnetic. But one of the most important effects we have in this limit is that the transverse pressure vanishes,

$$
p_{\perp}=-\Omega_{e}-B \mathcal{M}_{e}=0 .
$$

The effect (3.4) is of pure quantum origin and it is easy to understand since all electrons are confined to the Landau ground state, and the quantum average of their transverse momentum vanish. If we consider a white dwarf star in which the predominating contribution to the pressure is from the electron gas, the vanishing of $p_{\perp}$ means that the gravitational pressure (of order $G M^{2} / R^{4}$ where $R$ is the geometric average radius of the star) cannot be compensated and an instability appears leading to a transverse collapse, i.e., the resulting object (a neutron star or a black hole) would be ellipsoidal, in this case stretched along the direction of the magnetic field, as a cigar (a more quantitative study of the problem would require solving the Einstein equations). It is interesting to find the critical conditions for the oc- 
currence of this confinement to the state $n=0$, and in consequence, for the collapse. We have,

$n_{\mu}=I\left(\frac{\mu_{e}^{2}-m^{2}}{2 e B}\right)=\frac{2 \pi^{4} N_{e}^{2}}{e^{3} B^{3}} \sim 4.75 \times 10^{-20} \frac{N_{e}^{2}}{B^{3}}$,

and the condition for $I(x)<1$ might be found in some astrophysical conditions, e.g., for $N_{e} \sim$ $10^{30}, B=3.36 \times 10^{13} \mathrm{G}$, it is enough that $B \sim$ $B_{c^{\prime}}$ to satisfy it. For densities of the order of neutron stars, where a background of electrons and protons exist, if $N_{e}=10^{39}$, the previous condition, if valid, would lead to $B>10^{19} \mathrm{G}$.

\section{Degenerate $W$ gas}

The $W$ population in the Landau ground state is significant if $d=\sqrt{M_{W}^{2}-e B} \leq T$. In the degenerate limit, e.g. for $\sqrt{M_{W}^{2}+e B} / T \gg 1$, one can neglect the contribution from excited Landau states and by taking only the $n=0$ term in (2. $\overline{1} \overline{1})$, one can approximate the first two terms, since the main contribution to the integrals comes from very small momenta,

$\mathcal{M}_{W}=-\frac{e T}{4 \pi} \sqrt{d^{2}-\mu_{W}^{2}}+\frac{e B T}{4 \pi} \frac{1}{\sqrt{d^{2}-\mu_{W}^{2}}}+\mathcal{M}_{0 W}$

The first term, is the diamagnetic contribution which vanishes as $T \rightarrow 0$. The third is the vacuum contribution, which is asymptotically

$$
\mathcal{M}_{0 W} \sim-\frac{2 \Omega_{0}}{B}-\frac{e M_{W}^{2}}{16 \pi^{2}} \ln \left(M_{W}^{2} / e B-1\right),
$$

whose most important term is the second one which contributes para- or ferromagnetically for $B>M^{2} / 2 e$, having a logarithmic divergence as $B \rightarrow B_{c}$. That term has a negative contribution to the transverse pressure of vacuum for fields in the interval $B_{c} / 2<B \leq B_{c}$. The first term of $\mathcal{M}_{0 W}$ contribute diamagnetically. But for $B \rightarrow B_{c}$ the dominant term in (4.1) is the second, which is also para- or ferromagnetic, having a stronger divergence (inverse square root) than the vacuum term. To have a more explicit form for $(4.1)$, one must write $\mu_{W}$ in terms of the charge density. When confined to the Landau ground state [i] and taking $N \geq 10^{39}, T \sim 10^{-8}$ ergs and $B \leq B_{c}$, one is left with

$$
\mathcal{M}_{W} \simeq \frac{e N_{W}}{2 d}
$$

The most important consequence is that the contribution of this magnetization to the transverse pressure of the $W$ gas would be negative (see $(\dot{2} . \overline{1} \overline{1})$ ), and if $\mathcal{M}_{W} B$ contributes more than the pressure of other species, (the partial pressure $p_{3}=\Omega_{W}$ even decreases as $B \rightarrow B_{c}$ ) an instability occurs since the total pressure would be negative. Thus, for stability (also to prevent $W$ decay), we must assume some background able to keep the total pressure $p_{\perp} \geq 0$.

The cases considered previously are a sort of condensation for fermions and bosons in the $n=0$ state, but some sort of Bose-Einstein condensation actually takes place [1] $\left.\overline{1}_{1}\right]$ for bosons. For small momentum and magnetic fields strong enough $B \sim B_{c}$, the term $1 / d$ dominates and the main contribution to the $W$ propagator comes

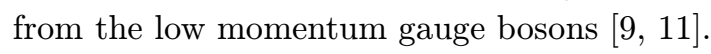

At any temperature, a spontaneous magnetization would appear in the condensate of charged bosons, say $W^{+}$, even at zero external field $H=$ $B-4 \pi \mathcal{M}=0$. This spontaneous magnetization could self-consistently maintain the microscopic field $B=4 \pi \mathcal{M}_{e, W}$.

\section{Self-consistent magnetization con- dition}

Let us assume the magnetization large enough to maintain the internal field $B$ self-consistently. Let us assume very large densities in the medium,

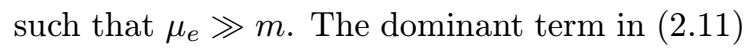
is $(4.2) \sim e N_{W} / 2 d$. At such field intensities $\overline{\mathcal{M}}_{W}$ diverges, but if we write the self-consistency condition for the $W$ sector, we have

$$
B=4 \pi \mathcal{M}=2 \pi \frac{e N_{W}}{d}
$$

Let us write $e B=x^{2} M_{W}^{2}$ and since $0 \leq x \leq 1$, we get easily the expression

$$
x^{2} \sqrt{1-x^{2}}=\frac{2 \pi e^{2} N_{W}}{M_{W}^{3}}=A .
$$

As $M_{W}^{3} / e^{2} \sim 10^{49} \mathrm{~cm}^{-3}$, even for $N_{W}$ exceeding largely the nuclear density, $A$ can be extremely small (For $A \sim 1, N_{W} \sim 10^{48} \mathrm{~cm}^{-3}$. For such densities, the horizon of events is $\sim 10^{-2} \mathrm{~cm}$ ). By writing $y=x^{2} \sqrt{1-x^{2}}$ we have a curve having an increasing branch starting from $x=0$ up 
to a maximum at $x_{M}=\sqrt{2 / 3}, y=A_{1}=2 \sqrt{3} / 9$, compatible with a density $N_{W} \sim 10^{48} \mathrm{~cm}^{-3}$. But the transverse collapse would have taken place at much lower densities: since the $W$ contribution to $\Omega$ is negligibly small, the pressure $p_{3}=$ $-\Omega$ comes essentially from the fermion (electron) background. From (3.5i), for $B \sim B_{c}$, the vanishing of $p_{\perp}$ takes place at $N_{e} \sim 10^{46} \mathrm{~cm}^{-3}$. We have also a decreasing branch from $x=x_{M}$ to $x=1$, compatible with densities smaller than $10^{48} \mathrm{~cm}^{-3}$. Thus, when solving $(5.21)$, which leads to a cubic equation, for $A>A_{1}$, we will not have real solutions. However, for $A \leq A_{1}$ we have two real positive solutions for $x$ ( coinciding for $\left.x^{2}=2 / 3\right)$. For $A \ll 1$, these solutions are $x_{1}=\sqrt{A+A^{2} / 2}$ and $x_{2}=\sqrt{1-A^{2}}$. The first solution means that $B$ increases with increasing $N_{W}$, (up to the value $B_{M}=2 M_{W} / 3 e$ ). In the second solution $B$ decreases as a function of $N_{W}$, its limit for $N_{W} \rightarrow 0$ being $B_{c}$. This obviously indicates that the expression for the magnetization must include the contribution from Landau states other than the ground state, which leads to a diamagnetic response to the field. This would compensate the increase of the self-consistent field with increasing $N_{W}$ to keep $B<B_{c}$.

This can be shown to occur from formula $\left(2 . \overline{1} \overline{1}_{-1}^{\prime}\right)$. If we name $N_{W g}$ the ground state density and $N_{W n}$ the density in other Landau states $\left(N_{W}=N_{W g}+\sum N_{W n}\right)$, for $B>B_{M}=2 B_{c} / 3$, $\partial B / \partial N_{W g}<0$ and $\partial B / \partial N_{W n}>0$ and excited Landau states start to be populated. The condensate in the ground state decreases in favor of the increase of the population in excited Landau states, which starts to grow and contribute diamagnetically to the total magnetization keeping it $\mathcal{M}=B<B_{c}$. But for the system reacting in this way, an enormous amount of energy (and angular momentum) would be required, of order respectively $N_{W} M_{W}$ and $N_{W}$ (Here we neglect the running of $M_{W}$ ).

\section{Conclusions}

We conclude, first, that if a degenerate electron gas is confined to its Landau ground state, its transverse pressure vanishes. This phenomenon establishes a limit to the magnetic fields expected to be observable in white dwarfs, and even in neutron stars. Second, that the instability of the vacuum in magnetic fields $B \sim B_{c}$, when it takes place in a hot and dense medium, is avoided, since either a transverse collapse is produced at fields of order $2 B_{c} / 3$, or else the self-consistent magnetization prevents the instability by a mechanism leading to a cooling of the system.

\section{Acknowledgments}

We would like to thank E. Nardi and W. Ponce for hospitality in Cartagena de Indias during the III SILAFAE.

\section{References}

[1] M. Chaichian, C. Montonen, S. Masood, A. Perez Martinez and H. Perez Rojas, Phys. Rev. Lett. 84 (2000), 5261.

[2] S. Chakrabarti, D. Bandyopadhyay and S. Pal, Phys. Rev. Lett. 78, 2898 (1997).

[3] K. Enqvist, P. Olesen and V. Semikoz, Phys. Rev. Lett. 69, 2157 (1992) .

[4] T. Vachaspati, Phys. Lett. B 265, 258 (1991).

[5] N.K. Nielsen and P. Olesen, Nucl. Phys. B 144, 376 (1978); J. Ambjørn, N.K. Nielsen and P. Olesen, Nucl. Phys. B 310, 625 (1988).

[6] J. Ambjørn and P. Olesen, Phys. Lett. B 214, 565 (1988); J. Ambjørn and P. Olesen, Nucl. Phys. B 315, 606 (1989).

[7] Landau, L. and Lifshitz, E., Statistical Physics, 3rd. Ed. Pergamon Press, Oxford (1980)

[8] A. Pérez Martínez, H. Pérez Rojas, A. Zepeda, Int. J. Mod. Phys. A 11, 5093 (1996).

[9] H. Pérez Rojas, Acta Phys. Pol. B 17, 861 (1986).

[10] Landau, L. and Lifshitz, E., The Classical Theory of Fields, Pergamon Press, Oxford (1965); H. Pérez Rojas, A. E. Shabad, Kratkie Soob. po Fizike (Lebedev Institute Reports, Allerton Press) 7, 16 (1976).

[11] H. Pérez Rojas, Phys. Lett. B 379, 148 (1996).

[12] S. L. Shapiro, S. A. Teukolski, Black Holes, White Dwarfs and Neutron Stars, J. Wiley and Sons, New York, (1983).

[13] K. Konno, T. Obata, and Y. Kojima, grqc/9910038. 